\title{
Instabilities in Luttinger liquids
}

\author{
D C Cabra ${ }^{1,2}$ and J E Drut ${ }^{1}$ \\ ${ }^{1}$ Departamento de Física, Universidad Nacional de la Plata, CC 67, (1900) La Plata, Argentina \\ ${ }^{2}$ Facultad de Ingeniería, Universidad Nacional de Lomas de Zamora, Cno de Cintura y Juan \\ XXIII, (1832), Lomas de Zamora, Argentina
}

Received 25 November 2002, in final form 4 February 2003

Published 24 February 2003

Online at stacks.iop.org/JPhysCM/15/1445

\begin{abstract}
We discuss the appearance of magnetic and charge instabilities, called respectively metamagnetism (MM) and phase separation (PS), in systems which can be described as perturbed Luttinger liquids. We argue that such instabilities can be associated with the vanishing of the effective Fermi velocity $v$, which in some cases coincides with a divergence of the effective Luttinger parameter $K$. We analyse in particular an $X X Z$ chain with next-nearestneighbour interactions in different limits where MM shows up and an extended Hubbard model where PS occurs. Qualitative agreement with previous studies is found.
\end{abstract}

(Some figures in this article are in colour only in the electronic version)

\section{Introduction}

The study of instabilities in low-dimensional strongly correlated electron systems has received much attention in the last few years. One of the main reasons is that a charge instability phenomenon (phase separation (PS)) often shows up in the vicinity of the superconducting transition in cuprates. In the case of double-exchange models for manganese oxides that exhibit the 'colossal'-magnetoresistance effect [1], this charge instability arises close to the transition to ferromagnetism and, interestingly, finite-size studies of both the two-dimensional realistic model and its one-dimensional version display similar features in this respect. The case of magnetic instabilities (metamagnetism (MM)) has also received attention recently in connection with the one-dimensional antiferromagnetic (AF) $X X Z$ spin chain with nextnearest-neighbour (NNN) interactions, where it was found that MM arises in a finite region of the phase space [2].

Generically, at the point where charge or magnetic instabilities occur, a divergent compressibility or magnetic susceptibility arises. This divergence is in turn associated with the coexistence of two phases with different hole concentrations or magnetizations.

The aim of this paper is to discuss a general way to determine whether an instability could show up in a given one-dimensional model, using Abelian bosonization. Our discussion applies 
to any one-dimensional model describable as an integrable model plus perturbations whose effect is to renormalize the Luttinger liquid (LL) parameters, $K$ and $v$. The effect of irrelevant operators is also discussed. Our discussion could also be relevant in the study of certain 2D systems which can realize the so-called sliding Luttinger phase [3], since a divergence in the 1D susceptibility leads to a singularity in the 2D one [4]. Generically, our approach provides a quick tool for studying the tendency of different perturbations to produce instabilities in the above-mentioned systems.

As a sample case for magnetic systems, we analyse the $X X Z$ chain with $\mathrm{NNN}$ exchange, treating first this last interaction perturbatively within a bosonization approach, and find qualitative agreement with the results obtained in [2] for finite systems (this problem was also studied in $[5,6])$. We also study the opposite coupling limit, which we call hereafter the 'zigzag' limit, where the system can be reinterpreted as a two-leg zigzag ladder. In this case, we determine a region in the parameter space where MM occurs.

To analyse the case of charge instabilities we consider the charge sector of the Hubbard model at incommensurate fillings, perturbed by a nearest-neighbour density-density interaction $V$. In this case we find that for small Coulomb repulsion $U$ there is a region where the system phase separates which corresponds to negative values of $V$. A similar situation was encountered in previous studies of the extended Hubbard model at quarter-filling [7], where it was shown that PS occurs for negative $V$, though in that case the values of the couplings are out of the perturbative regime. In $[8,9]$ PS was found in the same model at half-filling, also in a regime of large couplings.

\section{General discussion}

We consider a generic situation in which the low-energy degrees of freedom (which could correspond to either charge or spin variables) are described as those of a Tomonaga-Luttinger liquid in the unperturbed case. The corresponding Hamiltonian, once interactions are taken into account, will generically be of the form (modulo irrelevant terms)

$$
H_{0}+\int \mathrm{d} x\left(\alpha\left(\partial_{x} \phi\right)^{2}+\beta\left(\partial_{x} \tilde{\phi}\right)^{2}+\lambda\left(\partial_{x} \phi\right)\right)
$$

where $H_{0}$ corresponds to the Tomonaga-Luttinger Hamiltonian

$$
H_{0}=\frac{1}{2} \int \mathrm{d} x\left(v K\left(\partial_{x} \tilde{\phi}\right)^{2}+\frac{v}{K}\left(\partial_{x} \phi\right)^{2}\right) .
$$

The first two terms can be readily absorbed into a redefinition of the LL parameters, $K \rightarrow K_{e f f}$ and $v \rightarrow v_{e f f}$, while the third one changes the chemical potential in the case of charge variables and the magnetic field in the spin case.

Under the above-mentioned conditions the compressibility for the charge modes, described as those of an effective LL with parameters $K_{c}$ and $v_{c}$, can be shown to be given by [10]

$$
\kappa \propto \frac{K_{c}}{v_{c}},
$$

and, similarly, the magnetic susceptibility for the spin modes described as those of an effective LL with parameters $K_{s}$ and $v_{s}$ is given by

$$
\chi \propto \frac{K_{s}}{v_{s}} .
$$

It should be stressed that these expressions are valid at low energies, where the LL description holds, and for small magnetic fields, so one can treat the charge and spin degrees 
of freedom as independent. For higher fields one has to modify these expressions to take into account charge and spin mixing. (See the related papers [11] for the zero-field case and [12, 13] for non-zero field.)

One readily observes that a divergence in these quantities arises when either $K_{(c, s)}^{-1}$ or $v_{(c, s)}$ vanishes. It should be pointed out that these two things could happen simultaneously, but this is not always the case, and hence PS or MM instabilities are to be identified with the vanishing of the effective velocity. We argue that this is true, provided that the quantities $K v$ and $K / v$ remain positive definite and irrelevant perturbations do not change the large-scale behaviour. In [14] an attempt to characterize these instabilities in one-dimensional systems exhibiting a universal character described by a Tomonaga-Luttinger model was made. In this paper the authors identified the divergence of the above-mentioned thermodynamic quantities with a divergence in the so-called Luttinger parameter $K$. Although this interpretation led to a consistent analysis for the cases studied in [14], we argue that a more general criterion consists in identifying the instability regions with those where the velocity vanishes. This last statement can be understood as follows: by analogy with the unperturbed $X X Z$ chain in a magnetic field, we see that when the Fermi velocity goes to zero, we approach a ground state of FM nature (and $K$ does not necessarily diverge, though this happens for the particular case of the $X X Z$ chain for $\Delta=-1$ and zero field [14]). Then the magnetic susceptibility diverges as $1 / v_{\text {eff }}$ and, if this happens before reaching saturation, the magnetization curve as a function of the applied magnetic field presents a jump. Once we have shown that there is a jump in the magnetization curve, for some critical value of the applied magnetic field, $h_{c}$, we can conclude that two different values of the magnetization will coexist at this point [15].

In the next two sections we study two sample cases where this general discussion applies, the $X X Z$ chain with NNN interactions where MM has been shown to occur $[2,5]$ and the extended Hubbard model with nearest-neighbour interactions $V$ where PS appears for negative $V$ [7-9].

\section{The $X X Z$ AF chain with NNN interactions}

The Hamiltonian of the $X X Z$ AF with NNN interactions in a magnetic field is given by

$H_{X X Z}=J \sum_{i=1}^{N}\left(S_{i}^{x} S_{i+1}^{x}+S_{i}^{y} S_{i+1}^{y}+\Delta S_{i}^{z} S_{i+1}^{z}\right)+J^{\prime} \sum_{i=1}^{N}\left(S_{i}^{x} S_{i+2}^{x}+S_{i}^{y} S_{i+2}^{y}+\Delta S_{i}^{z} S_{i+2}^{z}\right)-h \sum_{i=1}^{N} S_{i}^{z}$.

The large-scale behaviour of the $X X Z$ chain can be described by a $U(1)$ free boson theory with Hamiltonian (2). The field $\phi^{i}$ and its dual $\tilde{\phi}^{i}$ are given by the sum and difference of the light-cone components, respectively. The constant $K$ governs the conformal dimensions of the bosonic vertex operators and can be obtained exactly from the Bethe ansatz solution for the $X X Z$ chain (see e.g. [16] for a detailed summary). We have $K=1$ for the $S U$ (2) symmetric case $(\Delta=1)$ and this is related to the radius $R$ of [16] by $K^{-1}=2 \pi R^{2}$. In (2) $v$ corresponds to the Fermi velocity of the fundamental excitations of the system.

In terms of these fields, the spin operators read

$$
\begin{aligned}
& S_{x}^{z} \sim \frac{1}{\sqrt{2 \pi}} \partial_{x} \phi+a: \cos \left(2 k_{F} x+\sqrt{2 \pi} \phi\right):+\frac{\langle M\rangle}{2}, \\
& S_{x}^{ \pm} \sim(-1)^{x}: \mathrm{e}^{ \pm \mathrm{i} \sqrt{2 \pi} \tilde{\phi}}\left(b \cos \left(2 k_{F} x+\sqrt{2 \pi} \phi\right)+c\right):,
\end{aligned}
$$

where the colons denote normal ordering with respect to the ground state with magnetization $\langle M\rangle$. The Fermi momentum $k_{F}$ is related to the magnetization of the chain as $k_{F}=$ 
$(1-\langle M\rangle) \pi / 2$. The effect of an $X X Z$ anisotropy and/or the external magnetic field is then to modify the scaling dimensions of the physical fields through $K$ and the commensurability properties of the spin operators, as can be seen from (6), (7). The constants $a, b$, and $c$ were numerically computed in the case of zero magnetic field [17] (see also [18]).

In what follows we study both the weak-coupling $\left(J^{\prime} / J \ll 1\right)$ and the zigzag $\left(J^{\prime} / J \gg 1\right)$ limits.

(i) The $J^{\prime} / J \ll 1$ limit

Using (6) and (7) the NNN interaction term in the bosonic language reads

$$
H_{N N N}=\alpha \int \mathrm{d} x\left(g_{1}\left(\partial_{x} \phi\right)^{2}+g_{2}\left(\partial_{x} \tilde{\phi}\right)^{2}+\lambda \partial_{x} \phi+\lambda^{\prime} \cos (\sqrt{8 \pi} \phi)\right),
$$

where $\alpha=J^{\prime} / J$ and $g_{1,2}, \lambda$, and $\lambda^{\prime}$ depend on $\Delta$ and the non-universal constants $a, b$, and $c$ as

$$
\begin{aligned}
& g_{1}=\Delta\left(\frac{1}{2 \pi}-2 \pi a^{2} \cos \left(4 k_{F}\right)\right)+2 \pi b^{2} \cos \left(4 k_{F}\right), \\
& g_{2}=-4 \pi\left(c^{2}-\frac{b^{2}}{2} \cos \left(4 k_{F}\right)\right)
\end{aligned}
$$

and

$$
\lambda=\frac{1}{\sqrt{2 \pi}} \alpha \Delta\langle M\rangle, \quad \lambda^{\prime}=\alpha b c .
$$

The first two terms in (8) have the effect of renormalizing both the compactification radius and the Fermi velocity in the following way:

$$
v_{e f f}^{2}=\left(v / K+2 \alpha g_{1}\right)\left(v K+2 \alpha g_{2}\right), \quad K_{e f f}^{2}=\frac{v K+2 \alpha g_{2}}{v / K+2 \alpha g_{1}},
$$

Now we can make contact with the analysis of [2]: the MM region is identified within our approach as the set of phase space points in which the susceptibility diverges for $0<M<M_{\text {sat }}$.

One immediately observes from (12) that, for zero magnetic field and without taking into account the effect of the $\lambda^{\prime}$-perturbation, both $v_{\text {eff }}$ and $K_{\text {eff }}^{-1}$ vanish simultaneously and that this happens when $\left(v / K+2 \alpha g_{1}\right)=0$ (see figure 1). However, this situation can change for two reasons: first, an extra term arises from the NNN perturbation, which renormalizes the external magnetic field $h$ and hence changes both $v_{\text {eff }}$ and $K_{\text {eff }}$ through the Bethe ansatz equations, generically in a way which removes the above-mentioned simultaneity. Second, if one goes beyond the zero-loop order, both $K_{\text {eff }}$ and $v_{\text {eff }}$ will renormalize in a different way due to the $\lambda^{\prime}$-term [19]. We will discuss this issue again in the context of charge instabilities in the next section. In particular, the boundary between the MM and the FM phases is obtained as the set of points at which $v_{\text {eff }}$ and/or $K_{\text {eff }}^{-1}$ vanish/es for $h=0$, and that between the MM and the AF phases as the set of points at which $v_{\text {eff }} \neq 0$ and $K_{e f f}^{-1} \neq 0$ for all values of the magnetization $\langle M\rangle<M_{\text {sat }}$.

Let us focus on the MM-FM boundary, which is easily obtained using (4) and (12). In this case, we can use the numerical values for the non-universal constants $a, b$, and $c$ appearing in (6) and (7) obtained in [17]. The boundary obtained in this way agrees qualitatively with that obtained in [2] (see figure 1). The lack of quantitative agreement is presumably due to the perturbative treatment of the NNN interactions, which could be improved by considering higher-loop contributions in a renormalization group analysis from the bosonization side and due to finite-size effects from the numerical one. However, our main aim is to discuss the appearance of instabilities in a generic and simple way. 


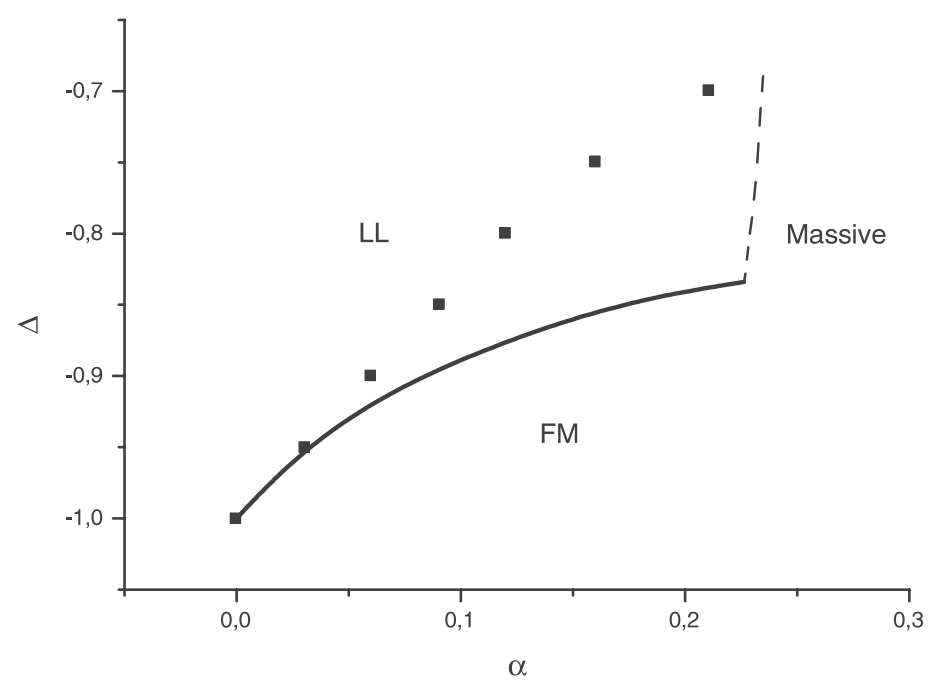

Figure 1. The ground state phase diagram for the $X X Z$ chain with $\mathrm{NNN}$ interactions in the limit $J^{\prime} / J \ll 1$ showing the lines of transition between the LL regime and the ferromagnetic one. Square dots are reproduced from [2] and the solid black line corresponds to our theoretical prediction. The dashed line indicates where the leading irrelevant operator becomes marginal.

One should be careful also about the regime of validity of this approach, since due to the renormalization of the Luttinger parameter $K_{\text {eff }}$, the scaling dimensions of the many discarded irrelevant perturbations change, and nothing prevents one of these from becoming relevant. In fact, by analysing the scaling dimension of the leading irrelevant $\lambda^{\prime}$-perturbation, we observe that it reaches the limiting value 2 at $\alpha_{c}=\frac{v\left(K^{2}-1\right)}{2 K\left(g_{1}-g_{2}\right)}$, and hence our approach ceases to be valid for $\alpha \geqslant \alpha_{c}$. This critical line separates the massless regime from a massive one, and our results compare well, qualitatively, with those obtained in [20] (the dashed line in figure 1). The study of the AF-MM boundary is in this case more involved due to the appearance of the non-universal constants $a, b$, and $c$ in the bosonized operators which are not available for non-zero magnetic field. (The estimation of the field dependence of these constants for $0 \leqslant \Delta<1$ has been carried out in [21] but the evaluation is more delicate on the ferromagnetic side $\Delta<0$ [22].)

\section{(ii) The zigzag limit $\left(J^{\prime} / J \gg 1\right)$}

In this limit we reinterpret the Hamiltonian (5) as a two-leg zigzag ladder in the weak-interchaincoupling limit [23-28]. In this description one represents each of the chains by one free compactified $U$ (1) boson. Thus, the whole ladder is represented by two bosons $\phi_{1}$, $\phi_{2}$, each governed by an action given by (2), plus the perturbative terms arising from the interchain zigzag coupling.

This system, in the presence of an external magnetic field, has been studied in [28], where it was shown that for non-zero magnetization a relevant interaction gives a mass to the 'relative' field $\phi_{r e l} \equiv\left(\phi_{1}-\phi_{2}\right) / 2$, while the 'diagonal' field, $\phi_{\text {diag }} \equiv\left(\phi_{1}+\phi_{2}\right) / 2$, remains massless.

The effective Hamiltonian governing the large-scale behaviour of $\phi_{\text {diag }}$ is then given by

$$
H_{e f f}=\frac{1}{2} \int \mathrm{d} x\left(v_{e f f} K_{e f f}\left(\partial_{x} \tilde{\phi}_{\text {diag }}\right)^{2}+\frac{v_{e f f}}{K_{e f f}}\left(\partial_{x} \phi_{\text {diag }}\right)^{2}+\lambda \partial_{x} \phi_{\text {diag }}\right),
$$




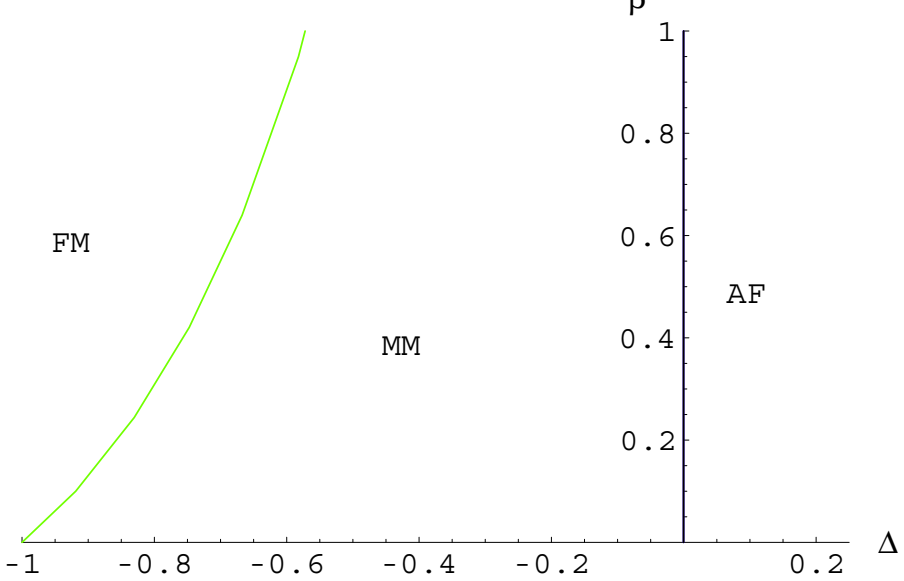

Figure 2. The qualitative ground state phase diagram for the $X X Z$ chain with NNN interactions in the limit $J^{\prime} / J \gg 1$.

where, using the notation $\beta=1 / \alpha, \lambda=\beta \Delta\langle M\rangle / \sqrt{2 \pi}$ and $v_{\text {eff }}$ and $K_{\text {eff }}$ are given by

$$
v_{e f f}^{2}=v^{2}\left(1+\frac{\beta \Delta K}{\pi v}\right), \quad K_{e f f}^{2}=K^{2}\left(1+\frac{\beta \Delta K}{\pi v}\right)^{-1} .
$$

There is however an extra term that mixes the fields $\phi_{\text {rel }}$ and $\phi_{\text {diag }}$ :

$$
H_{\text {mix }}=-\lambda^{\prime} \int \mathrm{d} x \partial_{x} \tilde{\phi}_{\text {diag }} \sin \left(\sqrt{4 \pi} \tilde{\phi}_{\text {rel }}\right) \text {. }
$$

The effect of this term was studied in [26] where it was shown that it gives rise to a spin nematic phase close to $\Delta=0$. The main point for our analysis is that the diagonal field is still described as that of a LL, and we expect the same picture to apply for $-1<\Delta \leqslant 0$. For this reason, we do not take into account this last term in what follows.

One then observes from (14) that the non-universal constants do not appear in the perturbing terms, and hence we can obtain in this case both the FM-MM and MM-AF boundaries. The results are presented in figure 2 .

\section{The Hubbard model with nearest-neighbour interactions}

We consider now the Hubbard model, defined as

$$
H=-\frac{1}{2} \sum_{j, \alpha}\left(\psi_{j, \alpha}^{\dagger} \psi_{j+1, \alpha}+\text { h.c. }\right)+U \sum_{j} n_{j, \uparrow} n_{j, \downarrow}+\mu \sum_{j, \alpha} n_{j, \alpha},
$$

where

$$
n_{j, \alpha}=\psi_{j, \alpha}^{\dagger} \psi_{j, \alpha} .
$$

In the absence of an external magnetic field it has been shown that this model presents charge-spin separation. It has also been shown (cf [29]) that the large-scale behaviour of the spin and charge degrees of freedom can be described by two decoupled boson field theories with dynamics governed by the Tomonaga-Luttinger Hamiltonian (note that the sign of the chemical potential is different to that in [29]). The parameters $K$ and $v$ can in each case be exactly obtained for all values of $\mu$ and $U$ via numerical solution of the Bethe ansatz equations 
in [30]. Approximate expressions for the velocity of the charge sector in the small- and large- $U$ regimes are given in [29] and a more general analysis can be found in [31].

The addition of a density-density interaction between nearest neighbours leads to the so-called extended Hubbard model, whose Hamiltonian is given by (HubHam) plus the term

$$
\delta H=V \sum_{j} n_{j} n_{j+1},
$$

where

$$
n_{j}=n_{j, \uparrow}+n_{j, \downarrow}
$$

It is a simple matter to show that for small $V$, modulo irrelevant operators, the effect of the perturbation (18) is to renormalize the parameters $\mu$ and $U$ as follows:

$$
\mu \rightarrow \mu+V, \quad U \rightarrow U+2 V .
$$

Therefore, the low-energy behaviour of the charge sector of the extended Hubbard model is that of a LL with parameters

$$
K_{\text {eff }}=K(U+2 V, \mu+V), \quad v_{\text {eff }}=v(U+2 V, \mu+V),
$$

where $K(U, \mu)$ and $v(U, \mu)$ are given by the exact Bethe ansatz solution of the non-extended Hubbard model (16). The above expressions are valid for arbitrary $U$ and $\mu$ but small $V$. This allows the determination of the divergences in the compressibility $\kappa$, as given by (3), which we identify with the zeros of the effective velocity.

We have only considered small values of $V$, since our approach is a perturbative one. Within this restriction, we have found no singularities, and therefore no PS, in the large- $U$ regime. On the other hand, as regards the region of small $U, V$, in which the effective velocity of the charge sector is given by

$$
v_{e f f}=v(U+2 V, \mu+V) \sim \pi(\mu+V)+\frac{U+2 V}{2 \pi},
$$

we have found from the roots of the above expression that PS occurs for negative values of $V$, similarly to what was found in [7-9], though for different fillings and coupling values. It should be stressed that $K_{\text {eff }}$ remains finite in the region where (22) vanishes, at this order in the couplings. If one includes, within the Luttinger picture, the effects of the quadratic terms in a perturbative manner (as we did in the previous section for the $X X Z$ model), one also encounters a simultaneous vanishing of the velocity and the inverse of the Luttinger parameter. We expect this effect to disappear on including higher-order contributions. Note also that we have chosen the value $\mu=0$ to correspond to full filling $(n=2)$; hence the Fermi velocity at $\mu=0, U=0, V=0$ vanishes. In figure 3 we plot the lines corresponding to the zeros of equation (22) in the limit of small $U, V$, in the $(\mu, U)$ plane for different values of $V$. The PS region can be located in the $(U, V)$ plane for a fixed filling for $U<-2 \pi^{2}(\mu+V)-2 V(V<0)$.

Following this approach, one could also study the appearance of instabilities in the magnetic sector and compare with the results obtained in [13].

A similar problem was studied in [32] and more recently in [33] where one-dimensional electrons interacting with phonons were considered. In these systems phonons could induce effective attractive interactions, and it was shown that under certain circumstances PS could occur and in principle be observed experimentally in thin carbon nanotubes [33].

\section{Conclusions and discussion}

We have discussed the appearance of MM and PS in systems that can be described as perturbed LLs. Specifically, we have studied the $X X Z$ spin chain with NNN interactions and the extended 


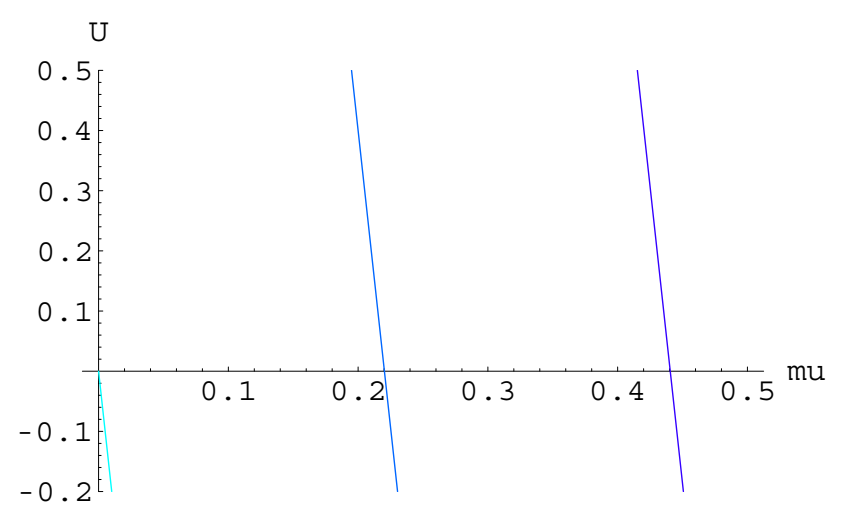

Figure 3. PS lines in the extended Hubbard model in the limit of small $U, V$, for $V=0,-0.2,-0.4$ from left to right.

Hubbard model with nearest-neighbour density-density interactions. We have found FM, AF, and $\mathrm{MM}$ regions in the zigzag limit of the $X X Z \mathrm{NNN}$ spin chain. In the weak-coupling limit, qualitative agreement with previous results was found for the MM-FM transition. We were not able to complete our search for MM due to lack of specific numerical data, an issue to be discussed elsewhere. The instabilities were in all cases identified as the roots of the inverse susceptibility (4).

Concerning the extended Hubbard model, according to our analysis, it does not present PS in the large- $U$ and small- $V$ limit. On the other hand, in the limit of small $U, V$, instabilities do show up, through the roots of the effective Fermi velocity (22), while the effective Luttinger parameter remains finite at first order in the couplings.

We argue that all the instabilities studied are associated in general with a vanishing effective velocity $v_{e f f}$. In fact, it is the roots of $v_{\text {eff }}$ that give a divergent compressibility of the extended Hubbard model, though, in the other cases studied, this coincides with a divergence of the effective Luttinger parameter $K_{e f f}$. This should not be regarded as the actual hallmark of the instabilities: although our treatment of the $X X Z$ NNN spin chain gives at the same time $v_{e f f}=K_{e f f}^{-1}=0$, this is only true in the absence of an external magnetic field and moreover ceases to be valid beyond the zero-loop order since the two parameters renormalize in different manners [19]. It should be stressed that our approach gives a simple way to qualitatively analyse instabilities in generic charge and magnetic systems provided that they can be described as perturbed LLs. Hence, it provides a quick tool for studying whether different perturbations could produce such instabilities.

\section{Acknowledgments}

It is a pleasure to acknowledge fruitful discussions with M Arlego, E Dagotto, A Furusaki, M D Grynberg, T Hikihara, A Honecker and P Pujol. The authors acknowledge partial financial support from CONICET and Fundación Antorchas.

\section{References}

[1] Moreo A, Yunoki S and Dagotto E 1999 Science 2832034 Dagotto E, Hotta T and Moreo A 2001 Phys. Rep. 3441

[2] Gerhardt C, Mütter K-H and Kröger H 1998 Phys. Rev. B 5711504 
[3] Kivelson S A, Emery V J and Fradkin E 1998 Nature 393550 Emery V J et al 2000 Phys. Rev. Lett. 852160

Vishwanath A and Carpentier D 2001 Phys. Rev. Lett. 86676

[4] Starykh O A, Singh R R P and Levine G C 2001 Preprint cond-mat/0106260

[5] Aligia A A 2000 Phys. Rev. B 63014402

Somma R D and Aligia A A 2001 Phys. Rev. B 64024410

Hirata S 1999 Preprint cond-mat/9912066

[6] Vieira V R, Guihéry N, Rodriguez J P and Sacramento P D 2001 Phys. Rev. B 63224417

[7] Penc K and Mila F 1994 Phys. Rev. B 499670

[8] Lin H Q and Hirsch J E 1986 Phys. Rev. B 338155

[9] Voit J 1992 Phys. Rev. B 454027

[10] Frahm H and Korepin V E 1990 Phys. Rev. B 4210553

Frahm H and Korepin V E 1991 Phys. Rev. B 435653

[11] Shiba H 1972 Phys. Rev. B 6930

Usuki T, Kawakami N and Okiji A 1989 Phys. Lett. A 135476

[12] Carmelo J M P, Horsch P and Ovchinnikov A A 1992 Phys. Rev. B 457899

Carmelo J M P, Horsch P, Campbell D K and Castro Neto A H 1993 Phys. Rev. B 48 RC4200

[13] Carmelo J M P, Guinea F and Sacramento P D 1997 Phys. Rev. B 557565

[14] Nakamura M and Nomura K 1997 Phys. Rev. B 5612840

[15] Dagotto E 1994 Rev. Mod. Phys. 66763

[16] Cabra D C, Honecker A and Pujol P 1997 Phys. Rev. Lett. 795126

Cabra D C, Honecker A and Pujol P 1998 Phys. Rev. B 586241 and references therein

[17] Hikihara T and Furusaki A 1998 Phys. Rev. B 58 R583

[18] Lukyanov S 1998 Nucl. Phys. B 522533

Lukyanov S 1999 Phys. Rev. B 5911163

Affleck I 1998 J. Phys. A: Math. Gen. 314573

[19] Giamarchi T 1990 Phys. Rev. B 442905

[20] Hirata S and Nomura K 2000 Phys. Rev. B 619453

[21] Hikihara T and Furusaki A 2001 Phys. Rev. B 63134438

[22] Hikihara T and Furusaki A 2001 private communication

[23] Chitra R, Pati S K, Krishnamurthy H R, Sen D and Ramasesha S 1995 Phys. Rev. B 526581

[24] White S R and Affleck I 1996 Phys. Rev. B 549862

[25] Allen D and Sénéchal D 1997 Phys. Rev. B 55299

[26] Nersesyan A A, Gogolin A O and Eßler F H L 1998 Phys. Rev. Lett. 81910

[27] Sørensen E, Affleck I, Augier D and Poilblanc D 1998 Phys. Rev. B 58 R14701

[28] Cabra D C, Honecker A and Pujol P 2000 Eur. Phys. J. B 1355

[29] Penc K and Sólyom J 1993 Phys. Rev. B 476273

[30] Lieb E and Wu F Y 1968 Phys. Rev. Lett. 201445

[31] Carmelo J M P, Horsch P, Bares P A and Ovchinnikov A A 1991 Phys. Rev. B 449967

[32] Loss D and Martin T 1994 Phys. Rev. B 5012160

[33] De Martino A and Egger R 2002 Preprint cond-mat/0212412 